unterschiedliche Erwartungen an das Social Web haben und dass ihr Umgang damit mit verschiedenen Risiken behaftet ist. Potenzielle Risiken und real erlebte Gefahren wurden in den qualitativen Interviews ausführlich thematisiert. Die Auswertungen der Interviews lassen eine Gruppierung in sechs verschiedene Handlungstypen zu. Die jeweiligen Zuordnungen stehen in engem Zusammenhang mit der Selbst-, Sozial- und Sachauseinandersetzung Jugendlicher im Alltag. Demzufolge variiert die Nutzungsintensität, die Art der Selbstdarstellung, das Beziehungsmanagement und die Informationssuche danach, welche Bedürfnisse und Interessen im Alltag aktuell bedeutsam sind und welche Entwicklungsbelange gerade bearbeitet werden. Wenig überraschend ist der Befund, dass das Internet sowohl der öffentlichen als auch der interpersonellen Kommunikation dient, wobei die Autoren und Autorinnen der Studie betonen, dass persönliche Treffen immer noch als die ideale Kommunikationsform für die meisten Anliegen erachtet wird. Onlinekommunikation wird als komplementärer Modus der Verständigung eingeschätzt. Der Band umfasst insgesamt neun Kapitel und schließt mit einem komprimierten Fazit und einer Zusammenstellung der Chancen von Partizipation und Mitbestimmung sowie den sozialen, ethischen sowie rechtlichen Problematiken, die sich im Umgang mit dem Social Web ergeben (können). Die Autoren und Autorinnen lassen es sich zum Schluss nicht nehmen, im Hinblick auf eine als notwendig erachtete $\mathrm{Me}-$ dienkompetenzförderung einige konkrete Handlungskonzepte in Bezug auf die Besonderheiten der Internetnutzung anzuregen. Diese scheinen vor dem Hintergrund einer sich sukzessive etablierenden Prosumentenkultur, die klassische Medienkompetenzmodelle auf den Prüfstand stellt, auch als gerechtfertigt.

Das Buch dokumentiert die anspruchsvolle Untersuchungsanlage, die Datenerhebung und Datenauswertung der Teilstudien und stellt die Ergebnisse der Analysen in einen übergeordneten Forschungszusammenhang, der ein umfassendes Bild der vielfältigen Nutzungsweisen von Social Web-Angeboten aufzeigt. Der multiperspektivische Zugang zur Erforschung des sozialen Phänomens der Online-CommunityKommunikation überzeugt und auch die $\mathrm{Zu}-$ sammenführung der einzelnen Untersuchungsmodule kann trotz der komplexen Darstellung als durchaus gelungen bewertet werden. Sicherlich bietet das Datenmaterial noch weitere Möglichkeiten der Auswertung, aber die Dynamiken der Netzkulturen lassen solche um- fänglichen Daten vermutlich auch binnen kurzer Zeit veralten.

Dagmar Hoffmann

\section{Jan-Hinrik Schmidt}

\section{Das neue Netz}

Merkmale, Praktiken und Folgen des Web 2.0

Konstanz: UVK, 2009. - 214 S.

ISBN 978-3-86764-146-3

Wie rezensiert man ein Buch, was im ersten Jahr nach seinem Erscheinen bereits zu einer Art Standardwerk bei den Studierenden avanciert ist - mit, aber auch ohne entsprechende Empfehlung? Wie rezensiert man zudem ein Buch, welches bereits sehr bekannt ist, da sein Autor (Jan Schmidt) das Thema - Web 2.0 - nicht nur wissenschaftlich bearbeitet, sondern auch nutzt und somit eine weitgehende Besprechung des Buches auch in Web 2.0-Kreisen bereits während des Schreibens bewirkt hat? Meine Antwort darauf ist der Versuch, den bereits vorhandenen Erfolg zu erklären und einzuordnen.

Die erste (und sehr allgemeine) Begründung für den bisherigen Erfolg des Buches ist die Tatsache, dass es eine Lücke füllt. Trotz der Aktualität und Beliebtheit des Themas „Web 2.0" gibt es nach wie vor nicht große Mengen an Auseinandersetzungen damit - insbesondere nicht der ernst zu nehmenden wissenschaftlichen Art. Zugleich ist diese Auseinandersetzung - die fragt, was denn tatsächlich das eigentlich Neue an all dem ist - sehr zugänglich und gut lesbar. Das mag ein zweiter Grund für den Erfolg sein. Ein dritter - und in meinen Augen wichtigerer - Grund liegt in der theoretischen Reflexion Schmidts (die immer auch mit Beispielen oder empirischen Erkenntnissen begründet wird). Schmidt versucht (und das gelingt ihm zu großen Teilen auch sehr gut), spezifische theoretische Rahmungen für das Web 2.0 Phänomen als relevante zu setzen und dies auch anschaulich zu begründen. Dabei ist der Teil zu persönlichen Öffentlichkeiten sicherlich der, der am weitesten reicht (siehe unten).

Zur Struktur des Buches: Schmidt beginnt „Das neue Netz“ mit einem eher deskriptiven Teil zur Entwicklung des Web 2.0 und verschiedenen Gattungen bzw. Diensten. Darin bezieht er sich auch auf die Frage der Nutzer, d. h. die Verbreitung, aber auch die Art und Weise der Nutzung (er bezieht sich hier, wie auch im gesamten Buch, auf vorhandene Studien, die z. T. auch seine eigenen sind).

Auf dieser Beschreibung aufbauend entwickelt er einen praxistheoretischen Ansatz zur 
Analyse von Nutzungspraktiken. Dieser wiederum setzt sich zusammen aus den Schwerpunkten Regeln, Relationen und Code. Diese tauchen als Bezugspunkte in der folgenden Analyse auch immer wieder auf. Die zentralen Kapitel aber sind das vierte und fünfte Kapitel (die Schmidt selber als verschiedene Teile des Buches bezeichnet), in denen er zunächst - im vierten-zwischen Identitäts-, Beziehungs- und Informationsmanagement unterscheidet. Dies sind - neben den genannten Schwerpunkten die zentralen Achsen der Analyse bei Schmidt. Diese werden fortgeführt im fünften Kapitel, in dem die Idee der persönlichen Öffentlichkeit entwickelt wird (und diese wiederum in Beziehung zu Fragen der Privatsphäre gesetzt wird). Die persönliche Öffentlichkeit grenzt sich deutlich von anderen Formen $a b$, indem hier kommunikative Äußerungen persönlicher $\mathrm{Na}$ tur im Vordergrund stehen, welche vor allem in sozialen Netzwerken getätigt werden. Die gesellschaftsweite Relevanz steht hier nicht im Vordergrund.

Auf diese doch relativ weit reichende Annahme folgen zwei Kapitel, die noch einmal andere Schwerpunkte setzen: zum einem geht es um professionell hergestellte Öffentlichkeiten (Journalismus \& politische Kommunikation), die durch das Web 2.0 erweitert werden. Und es geht zum anderen um verschiedene Formen des Umgangs mit Information und Wissen (Wikipedia und Tagging). Als letztes dann fasst Schmidt seine Annahmen in einer kurzen Kritik des Web 2.0 zusammen.

Insgesamt also haben wir es bei „Das neue Netz" mit einem gut strukturierten, gut argumentierenden Buch mit anregenden theoretischen Verweisen zu tun. Schwierig waren nur ein paar Längen: Es gab eine nicht gerade kleine Menge an Erklärungen zu bzw. Auflistungen von spezifischen Anwendungen. Die Betonung der Nutzerperspektive hingegen (welche die Angebotsperspektive erweitern soll) tut dem Thema gut, da zugleich nicht primär mit Annahmen, sondern mit empirischen Daten argumentiert wird. Auch der Praxis-Begriff passt hier gut - wenngleich man nicht umhin kommt, ob der modischen Komponente des selbigen ein wenig zu schmunzeln. Schmidt aber begründet seine Wahl sehr fundiert. Etwas weniger überzeugt war ich persönlich von der Einordnung in Regeln, Code und Relationen. Obwohl sehr anregend formuliert und als theoretische Setzung vieles vereinend, was auch ich für relevant halte (von affordances über Konventionen zu Öffentlichkeiten), so war die Übersetzung in die Beispiele dabei etwas schwieriger bzw. wirkt die nochmalige Kopplung an Identitäts-,
Beziehungs- und Informationsmanagement dann manches Mal etwas konstruiert oder offensichtlich (z. B. wenn darauf verwiesen wird, dass die Software von Profilseiten eine Art standardisierter Selbstdarstellung erzwingt, die nicht immer den eigentlich Darstellungsbedürfnissen entspricht-vgl. S. 83). Eventuell ist diese Konstruktion aber auch eine, die sich in weiteren, spezifisch darauf aufbauenden Studien noch deutlicher profilieren wird. Bereits erwähnt wurde das Konstrukt der persönlichen Öffentlichkeiten als sehr hilfreiches, welches diesen wichtigen Bereich der Debatten zum neuen Netz sicherlich prägen wird. Die letztendliche Einordnung der Phänomene erfolgt angenehm nüchtern - der Gestaltungsspielraum des Social Web wird betont, ohne zu beschönigen oder zu mystifizieren. Auch zukünftigen Generationen von Studierenden (und Kollegen) werde ich dieses Buch somit empfehlen!

Maren Hartmann

\section{Siegfried J. Schmidt / Jörg Tropp (Hrsg.) \\ Die Moral der \\ Unternehmenskommunikation}

Lohnt es sich, gut zu sein?

Köln: Halem, 2009. - 408 S.

ISBN 978-3-938258-48-4

Empirisch belegt ist die Tatsache, dass Unternehmen, die gesellschaftliche Verantwortung übernehmen, also Corporate Social Responsability (CSR) praktizieren, langfristig wirtschaftlich erfolgreicher sind. Aber an diese Tatsache schließen sich eine Reihe von Fragen an. Wie tief muss die Selbstbindung an Werte und Normen gehen, um glaubhaft zu sein? Genügt es, soziales und Umwelt-Sponsoring zu praktizieren und davon zu reden, oder muss das beispielsweise auch Fairness gegenüber den eigenen Mitarbeitern einschließen? Stellen Geld und Moral nicht doch einen Widerspruch darzumindest für kritische Konsumenten oder wenn man den Absolutheitscharakter des Moralischen in Rechnung stellt?

Vor diesem Problemhintergrund vereinigt der Sammelband 26 Einzelartikel und ordnet sie in vier Kapitel: I. Markt, Reputation und Werte; II. Kommunikation und Moral; III. Werbung, Marke und Moral; IV. Empirische Befunde zu Moral und Unternehmenskommunikation. Geschrieben wurden die Artikel im Wirtschaftskrisen-Jahr 2008; eine Bezugnahme zum Ernst und dem globalen Charakter der Schief- 